\title{
PROPUESTA DE DISEÑO DE MOBILIARIO PARA LABORATORIOS INFORMÁTICOS DE LA FACULTAD DE INGENIERÍA DE LA UNIVERSIDAD DISTRITAL FRANCISCO JOSÉ DE CALDAS
}

\section{FURNITURE DESIGN PROPOSAL FOR COMPUTER LABS OF THE FACULTY OF ENGINEERIN OF THE UNIVERSITY DISTRITAL}

\begin{abstract}
RESUMEN
Este artículo muestra el diseño y desarrollo del mobiliario para los actuales equipos de cómputo ubicados en los laboratorios informáticos de la Universidad Distrital Francisco José de Caldas, en Bogotá, Colombia, con el fin de adaptarlo a estas herramientas tecnológicas y proporcionar una mejor distribución del espacio disponible. Para ello se analizaron las características antropométricas de los estudiantes, sus necesidades, aspectos relacionados con salud e higiene ocupacional, ergonomía y cumplimiento de normas NTC 4641, NTC 5655, NTC 4731 del mobiliario actual.
\end{abstract}

Posteriormente se empleó la metodología -“Despliegue de la función de calidad" (QFD, Quality Function Deployment) para determinar los principales parámetros con que debería contar el mobiliario y el proveedor con la opción más cercana a satisfacerlos. Después de caracterizar de una manera cuantitativa lo cualitativo, se procedió a desarrollar un proceso sistémico que generara elementos para concretar el diseño que une los elementos técnicos y los requisitos del cliente (usuario).

Por último, se realizaron varios diseños mediante el software Solid Edge. Se seleccionó un modelo que cumplía con los requisitos del cliente, arrojados por la herramienta QFD y otras metodologías de diseño, y que permitiera la optimización del espacio.

Palabras clave: despliegue de la función de calidad, ergonomía, higiene y salud ocupacional; diseño

\footnotetext{
ABSTRACT

This article shows the design and development of furniture for today's computer equipment located within the computer laboratories in the seat Sabio Caldas Distrital University, so that fits these technological tools and provide a better distribution of the available space. In this way, we intended to analyze the anthropometric characteristics of students, their needs, issues related to occupational health and hygiene, ergonomics and compliance NTC 4641, NTC 5655 NTC 4731 the cu-
}

Leonardo Emiro Contreras Bravo Ingeniería de Materiales y Procesos de Manufactura

Docente Universidad Distrital Francisco José de Caldas

lecontrerasb@udistrital.edu.co

Bogotá, Colombia

Giovanna Andrea Castillo Sánchez Estudiante Universidad Distrital Francisco José de Caldas castillogiovanna@hotmail.com Bogotá, Colombia

\section{Magda Lorena Rodríguez Casas} Estudiante Universidad Distrital Francisco José de Caldas mlorena.rc@hotmail.com Bogotá, Colombia

Tipo: Reporte de caso

Fecha de Recepción: Mayo 27 de 2014 Fecha de Aceptación: Octubre 20 de 2014 
rrent furniture.

Then, the methodology (QFD Quality Function -Deployment) was used in order to identify key parameters that must be the furniture, and the supplier with the closest option to satisfy them. After a quantitatively decipher the qualitative, we proceed to develop a systemic process that generates particular elements to reach the design concrete steps by joining the technical elements and requirements of the client (user).

In the final part, several designs using Solid Edge software are made. A model that meets customer requirements thrown by the QFD tool, other design methodologies is selected and allow optimization of space.

Keywords: quality function deployment, ergonomics, occupational health and hygiene, design

\section{INTRODUCCIÓN}

En la actualidad, un gran número de organizaciones se enfrentan al hecho de contar con personas desempeñando labores durante un gran número de horas frente a herramientas tecnológicas como lo son los computadores; esto surge como consecuencia del rápido desarrollo en el manejo de información y de las telecomunicaciones que han facilitado la realización y seguimiento de variados procesos, e incluso han llegado a complementar el aprendizaje, por lo que hoy en día su uso se ha convertido en una necesidad imperiosa. No obstante, su empleo prolongado genera la necesidad de una apropiada adaptación física para que la productividad y el rendimiento se mantengan en niveles altos y se eviten posteriores traumas físicos y psicológicos en los usuarios que afecten su desempeño.

La Universidad Distrital brinda sus programas de formación actualmente con equipos que fueron adquiridos hace alrededor de cuatro años, en mobiliario comprado con mayor tiempo de anterioridad (figura 1), de manera que no se adecua a las dimensiones de los equipos actuales, ni a las normas NTC 4641, NTC 5655, NTC 4731 que abarcan los principios para el dise- ño ergonómico de sistemas de trabajo y que establecen que el diseño del puesto de trabajo debe permitir tanto la estabilidad como la movilidad postural. Por ejemplo, como se aprecia en la figura 1, la estructura fija en donde se ubica el teclado limita la movilidad de las extremidades inferiores, lo que dificulta la correcta ejecución de las labores, y físicamente puede ocasionar graves problemas como tensión articular y muscular, al igual que venas várice; incluso, como lo afirma [1], el permanecer sentado comprime las venas, lo que dificulta la circulación de la sangre hacia los músculos en el momento en que estos más lo necesitan; así, en esta posición el aparato circulatorio disminuye su flujo, se reduce el ritmo cardíaco, por lo que para reducir riesgos de un mal flujo sanguíneo, la posición de modo sentado requiere que las piernas estén ubicadas en un ángulo igual o un poco mayor a $90^{\circ}$. Además, la estructura rígida de este mobiliario podría estar repercutiendo en un uso ineficiente del espacio de laboratorio de la Universidad de acuerdo con la NTC 4595.

De acuerdo con los aspectos anteriormente mencionados, este trabajo se enfocó en proponer el diseño del mobiliario para los actuales equipos de cómputo ubicados en los laboratorios informáticos de la sede Sabio Caldas de 
la Universidad Distrital, de tal manera que se adapte a las herramientas tecnológicas y que junto con la ergonomía y el diseño industrial posibilite darles las dimensiones adecuadas a aquellos elementos de trabajo de uso cotidiano del estudiantado [2], con el fin de proporcionar una mejor distribución del espacio disponible y cumplir con las normas técnicas mencionadas.

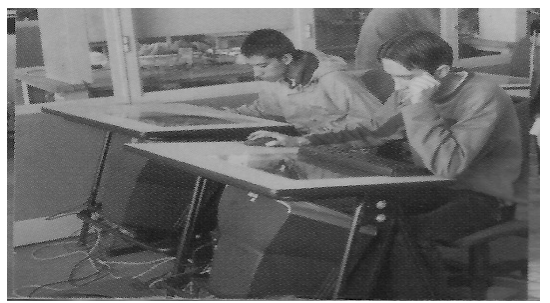

Figura 1a. Mobiliario actual para equipos de los laboratorios informáticos localizados en la sede Sabio Caldas

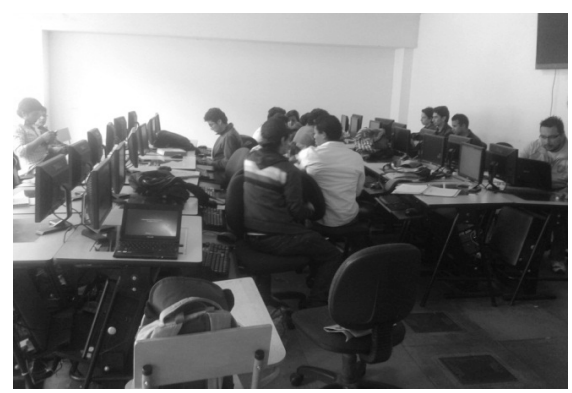

Figura 1b. Falta de adaptación de los usuarios con el mobiliario universitario de los salones informáticos

\section{GENERALIDADES DEL DESPLIEGUE DE LA FUNCIÓN DE CALIDAD (QFD)}

Para elaborar la propuesta de diseño del mobiliario se tomó como pauta el concepto básico de la metodología QFD, el cual permite planificar todo proceso, tomando como referencia las necesidades de los usuarios o la "voz del cliente", para satisfacer sus requerimientos en totalidad y ofrecerles, a su vez, siempre resultados de calidad [3]. De esta manera, las fases del diseño que se detallan a continuación están dirigidas a suplir las demandas de los usuarios del mobiliario.

\subsection{Estructura de QFD}

La analogía más usada para explicar cómo está estructurado el QFD es una casa [4]. La figura 2 muestra cómo se estructura una matriz QFD básica. El costado izquierdo, componente 1, es el input del cliente; en esta etapa se determinan los requerimientos del usuario en relación con el producto (qués). Para satisfacer estas necesidades, se evalúan ciertas especificaciones de desempeño, que conforman el techo interior de la casa o componente 2 (cómos). A continuación se analiza la relación existente entre estos aspectos técnicos, con lo que se conforma el techo de la casa (matriz de correlaciones) o componente 3. Así mismo se debe establecer el nivel de relación entre cada una de las necesidades referidas por el usuario con las características técnicas determinadas, presentado como componente 4 (matriz de relaciones entre cómos y qués) y para el cual se concede un valor numérico que represente dicha relación.

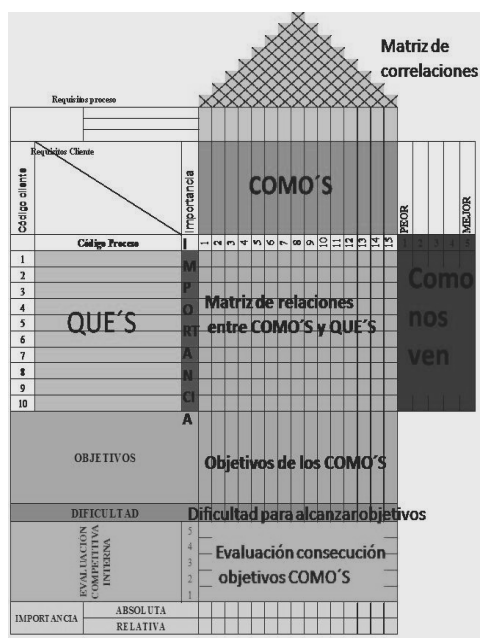

Figura 2. Estructura de la matriz QFD [5]

El fondo o base de la casa, componente 5 (objetivos de los cómos), es donde se jerarquizan los requisitos técnicos críticos del proceso. El componente 6 (cómo nos ven), a la derecha, pondera la satisfacción de las necesidades de los usuarios a partir de las propuestas presentadas por la competencia. El componente 7 se refiere a la relación entre las propuestas disponibles (tanto las de la competencia como la propia) y los componentes técnicos. Finalmente, el componente 8 (evaluación consecución de los objetivos cómos) arroja la propuesta más cercana al cumplimiento de los requerimientos técnicos. 


\subsection{El proceso QFD}

Un ciclo completo del proceso de QFD lo constituyen seis matrices similares a las de la figura anterior. La matriz 1 se usa para comparar los requerimientos del cliente con las características técnicas del producto. Todas las otras matrices se originan de esta primera matriz. La matriz 2 se usa para comparar las características técnicas en la matriz 1 con sus tecnologías aplicadas asociadas. Sirven para contestar preguntas como: ¿qué quiere el cliente?, ¿cuáles son los requisitos técnicos relacionados con las características que quiere el cliente? La matriz 3 se usa para comparar las tecnologías aplicadas de la matriz 2 con sus procesos de manufactura asociados. La matriz 4 se usa para comparar los procesos de manufactura de la matriz 3 con sus procesos de control de calidad asociados. La matriz 5 se usa para comparar los procesos de control de calidad con sus procesos de control estadístico del proceso. La matriz 6 se usa para comparar los parámetros del control estadístico del proceso con las especificaciones que se han desarrollado para el producto terminado.

\section{METODOLOGÍA}

\subsection{Diagnóstico}

Utilizando el método de la observación [6] y contrastándolo con la NTC 4641, que comprende las características con las que debe contar el mobiliario escolar, se lograron detectar algunas deficiencias (figura 3) en las dimensiones del mobiliario común de las 12 salas de laboratorios, tales como la altura de la superficie de trabajo, la profundidad y ancho del pupitre, la altura de la superficie del asiento y la longitud efectiva de la superficie del asiento, que impide a los usuarios adoptar posturas adecuadas, lo que dificulta la correcta ejecución de las labores, y puede ocasionar graves problemas como tensión articular y muscular [7].

Otros aspectos importante del mobiliario actual (no se enuncian todos los encontrados) son: a pesar de contar con un espacio amplio, la mesa no cuenta con un lugar específico para escribir o tomar apuntes (labor necesaria en el desarrollo de estas clases), lo que obliga al estudiante a alterar su posición y a adoptar una postura inadecuada, lejano a lo que establece la NTC 4731. En cuanto a la ubicación de la pantalla del computador, se ubica en un punto distante del campo visual del usuario, que no puede ser modificado debido a las conexiones eléctricas de la pantalla y que puede llegar a producir "gran fatiga muscular en las vértebras cervicales como consecuencia de la inclinación de la cabeza superior a un ángulo de $30^{\circ}$, sin dejar de lado que mantener el tronco inclinado hacia adelante, sin apoyo en el respaldo y los antebrazos en la mesa, genera una alta presión intervertebral en la zona lumbar, que podría ser la causa de un proceso degenerativo importante de la columna en esta zona" [1].

La distribución del espacio dentro de los laboratorios es de gran relevancia, ya que con base en ella se determina la capacidad de estudiantes que pueden hacer uso de la sala. De acuerdo con la NTC 4595, un laboratorio de informática se clasifica como un ambiente tipo C, para el cual se recomienda tener un área ocupada por estudiante de 2,3 a $2,5 \mathrm{~m}^{2}$. Se identificó que en la mayoría de salas, la disposición del mobiliario desperdicia gran espacio y varios usuarios no cuentan con la posibilidad de ver el tablero. Situaciones como éstas obligan a los usuarios a ingresar otro tipo de sillas a la sala, las cuales no se adaptan a la mesa de trabajo, ni a la tarea que se pretende llevar a cabo en estos lugares, ya que el estudiante queda situado lejos no sólo del tablero sino de los equipos, lo que disminuye su capacidad para visualizar las actividades que se están realizando.

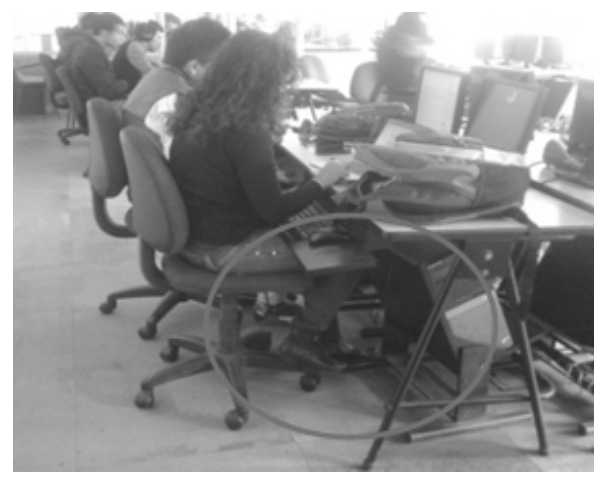

Figura 3a. Uso de la base de la silla como descansa pies

Propuesta de diseño de mobiliario para laboratorios informáticos de la Facultad de Ingeniería de la Universidad Distrital Francisco José de Caldas 


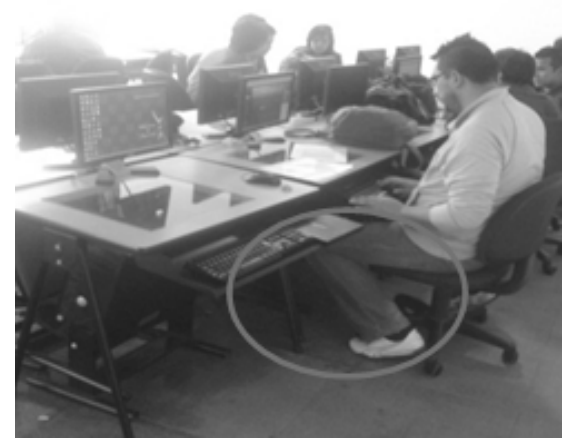

Figura 3b. Desajuste entre la mesa y silla de trabajo que limitan la movilidad de las extremidades inferiores del cuerpo de los usuarios

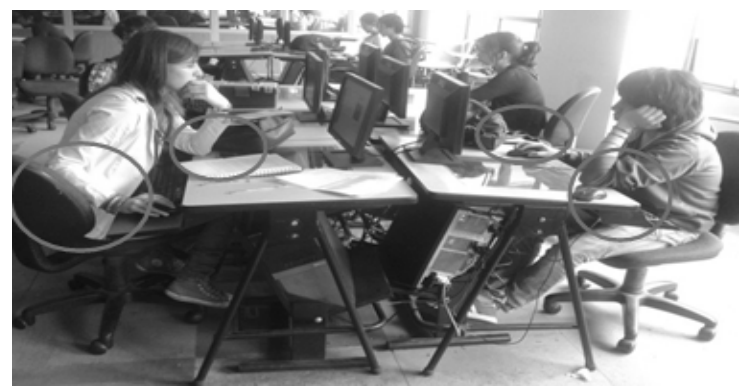

Figura 3c. Presión en el abdomen, desajuste en el uso del mouse y desajuste para la ejecución de la labor de tomar apuntes

\subsection{Captación de las demandas del cliente (voz del cliente)}

Uno de los métodos cualitativos utilizados para captar los requerimientos de los usuarios es la entrevista [5]. Por ello, se realizaron 32 entrevistas para captar las necesidades fundamentales de los usuarios [6] en relación con el uso del mobiliario de los laboratorios informáticos, ya que luego de esta cantidad no se revelaron nuevas necesidades. Las entrevistas incluyeron preguntas como: ¿Qué aspectos son los que más le gustan o considera positivos del mobiliario actual existente en los laboratorios informáticos? ¿Qué aspectos son los que no le gustan o considera negativos del mobiliario actual existente en los laboratorios informáticos? Si tuviera la oportunidad de realizar algún cambio o modificación al mobiliario, ¿qué aspectos tendría en cuenta?, ¿Qué modificaciones realizaría?
De acuerdo con el modelo planteado por [7], las demandas de los clientes pueden ser de tres clases: necesidades básicas, que son aquellas que el usuario siempre esperaría tener y le causarían insatisfacción en caso de no contar con ellas; necesidades explícitas, que son aquellas que entre mayor cumplimiento más satisfacción producirán en el usuario; y necesidades atractivas, que son fuente de sorpresa para el usuario y le generan valor agregado. Basados en ese modelo, se jerarquizaron las 75 necesidades previamente expresadas por los usuarios en estos tres grupos. Se considera que este es un número apropiado de necesidades, pues "no se debe hacer una lista excesiva de demandas del cliente y/o características de la calidad, ya que se tienden a difuminar los aspectos más importantes entre otros secundarios" [8].

\subsection{Características técnicas (CT)}

En cuanto a los factores antropométricos, se realizó un estudio basado en el análisis de las medidas de los estudiantes activos inscritos en la Facultad de Ingeniería que hacen uso de este mobiliario $(6,296$ estudiantes). Para el cálculo de la muestra (376 estudiantes) se tomaron en cuenta estudiantes en edades entre 16 y 25 años, periodo en el cual no se presentan grandes variaciones físicas de talla y peso [9]. Se tuvo en cuenta la fórmula estadística de [10], aplicable para una población finita (ecuación (1).

$$
\eta=\frac{N}{e^{2}(N-1)+1}
$$

Donde " $e$ " es el error de estimación (para el presente caso se supone un error del 5\% para un nivel de confianza del 95\%).

A partir de las deducciones antropométricas mostradas por [7], se obtuvieron las medidas principales que se emplearon en el diseño del mobiliario. De ellas cabe resaltar: altura al nivel de los ojos en posición sentado; altura al nivel de los codos en posición sentado, altura poplítea, anchura bideltoidea, distancia nalga-poplítea, anchura de la cadera, longitud codo-muñeca, longitud del pie y distancia espaldar-altura 
poplítea, entre otras. Hay que tener en cuenta que no sólo esta información fue tomada en el diseño, sino que también se tuvieron en cuenta el cumplimiento de las normas técnicas colombianas para el diseño final.

Luego se tomaron los parámetros del mobiliario dictados por la NTC 4641 y se contrastaron con las mediciones antropométricas mencionadas, para elaborar un listado de aquellas con las que debería contar el mobiliario. Esto, sumado con algunos aspectos ergonómicos y de salud e higiene ocupacional recomendados por [10], arrojó la lista de requerimientos técnicos a tenerse en cuenta más adelante.

\subsection{Estudio de proveedores}

Se eligieron los tres proveedores principales de mobiliarios adaptables a laboratorios de informática, según la clasificación de la Cámara de Comercio de Bogotá (la clasificación del 2011 era la información más reciente disponible), que muestra las empresas con mayores ventas en este sector. Estas son (figura 4): Muebles Carvajal: Línea Link (figura 4a), Muebles Solinoff: Proyecto Universidad Jorge Tadeo Lozano (figura 4b), Muebles Servex Colombia (figura 4c).

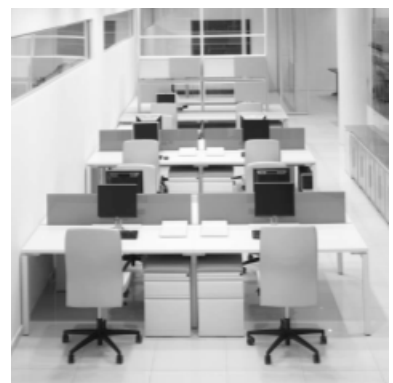

(a)

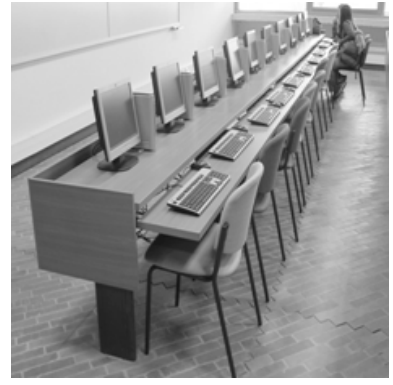

(b)

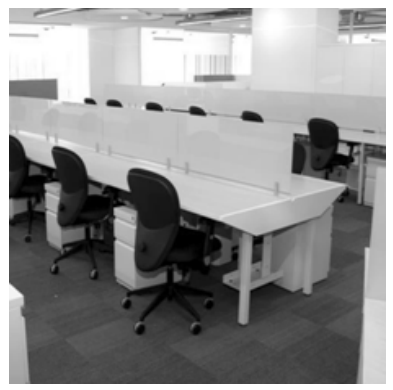

(c)

Figura 4. Propuestas de mobiliario de los proveedores con las mayores ventas en el 2011 [11-13]

\section{RESULTADOS Y ANÁLISIS DE RESULTADOS}

\subsection{Casa de la Calidad}

El primer resultado obtenido es la Casa de la Calidad; esta es una técnica que permite relacionar en una matriz los aspectos previamente hallados. En consecuencia, se organizó en ella la información inicialmente recolectada (figura 5).

En la parte izquierda de esta casa se exponen las 75 necesidades detectadas en los usuarios del mobiliario. En la parte superior se detallan las características técnicas con las que se podrían suplir las necesidades de los usuarios. Entre los dos aspectos se conformó una matriz en la que se pretende reflejar, desde luego, la relación entre los requerimientos de los usuarios y los aspectos técnicos que podrían contribuir a su satisfacción. A continuación se otorgaron unas valoraciones para medir el grado de relacionamiento entre ellas, de igual manera se utilizaron los símbolos "+" y “-" para evidenciar el tipo de vínculo existente entre cada uno de los requerimientos técnicos, indicando si éste era directa o inversamente proporcional.

Posteriormente se concedió una apreciación a las propuestas brindadas por cada uno de los proveedores acorde con la clasificación de las necesidades presentadas; y luego, a través de las ecuaciones (2) y (3), se dio una calificación para cada opción del mercado con el fin de hallar la mejor alternativa para suplir estos requerimientos, la cual fue la del mobiliario de

Propuesta de diseño de mobiliario para laboratorios informáticos de la Facultad de Ingeniería de la Universidad Distrital Francisco José de Caldas 
Servex Colombia.

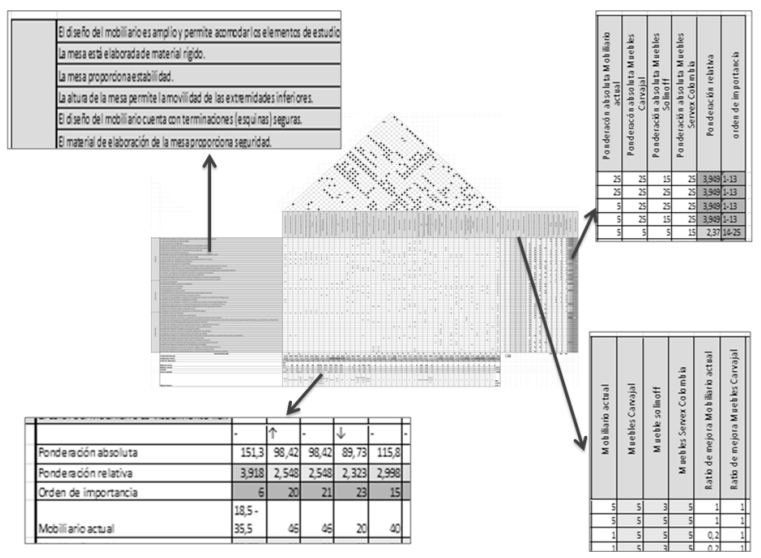

Figura 5. Casa de la calidad - QFD

$$
\begin{gathered}
R M=\frac{V S N P A}{V A C N} \\
P A N=V A C N \cdot V I N U \cdot R M
\end{gathered}
$$

Donde

$R M$ : Ratio de la mejora

VSNPA: Valoración de la satisfacción de la necesidad presentada actualmente

$V A C N$ : Valoración de un alto cumplimiento de la necesidad.

VINU: Valoración de la importancia de la necesidad para el usuario.

El QFD revela cuáles son las características técnicas que más influyen o pueden solucionar los requisitos del cliente. Esta primera matriz arrojó que correspondían a 36 características técnicas (CT) para este caso.

\subsection{Análisis morfológico}

Con la ayuda de un método analítico-combinatorio denominado análisis morfológico, cuyo objetivo es resolver problemas mediante el análisis de las partes que lo componen, se redujeron las características técnicas (de 36 a 13) y los requisitos del cliente (se redujeron de 75 a 9). El análisis realizado con ayuda de este método se muestra en la tabla 1.
Tabla 1. Variaciones de las características del diseño de

\begin{tabular}{|c|c|c|c|}
\hline Característica & $\begin{array}{l}\text { Variación } \\
\text { boceto } 1\end{array}$ & $\begin{array}{l}\text { Variación } \\
\text { boceto } 2\end{array}$ & $\begin{array}{l}\text { Variación } \\
\text { boceto } 3\end{array}$ \\
\hline $\begin{array}{c}\text { Soporte de la } \\
\text { mesa }\end{array}$ & $\begin{array}{l}\text { Soporte } \\
\text { angular }\end{array}$ & $\begin{array}{l}\text { Soporte } \\
\text { rectangular }\end{array}$ & $\begin{array}{l}\text { Soporte } \\
\text { rectangular } \\
\text { cruzado }\end{array}$ \\
\hline Portateclado & $\begin{array}{l}\text { Portateclado } \\
\text { móvil } \\
\text { inferior al } \\
\text { tamaño de } \\
\text { la mesa }\end{array}$ & $\begin{array}{c}\text { Portateclado } \\
\text { móvil del } \\
\text { ancho de la } \\
\text { mesa }\end{array}$ & $\begin{array}{c}\text { Portateclado } \\
\text { móvil inferior } \\
\text { al tamaño de } \\
\text { la mesa con } \\
\text { portamouse } \\
\text { desplegable }\end{array}$ \\
\hline Porta-CPU & $\begin{array}{l}\text { Porta-CPU } \\
\text { removible } \\
\text { ajustada a la } \\
\text { mesa (sólo } \\
\text { proporciona } \\
\text { soporte } \\
\text { inferiora la } \\
\text { CPU) }\end{array}$ & $\begin{array}{l}\text { Porta-CPU } \\
\text { ajustada a } \\
\text { un lado del } \\
\text { soporte de } \\
\text { la mesa }\end{array}$ & $\begin{array}{c}\text { Porta-CPU } \\
\text { removible } \\
\text { ajustada } \\
\text { a la mesa } \\
\text { (con soporte } \\
\text { lateral) }\end{array}$ \\
\hline Apoyapiés & $\begin{array}{l}\text { No cuenta } \\
\text { con } \\
\text { apoyapiés }\end{array}$ & $\begin{array}{c}\text { Apoyapiés } \\
\text { adicional al } \\
\text { mobiliario }\end{array}$ & $\begin{array}{c}\text { Apoyapiés } \\
\text { adaptado a } \\
\text { un soporte } \\
\text { de la mesa } \\
\text { adicional }\end{array}$ \\
\hline $\begin{array}{l}\text { Soporte al } \\
\text { final de la } \\
\text { mesa }\end{array}$ & Sin soporte & $\begin{array}{l}\text { Soporte en } \\
\text { vidrio }\end{array}$ & $\begin{array}{c}\text { Soporte en } \\
\text { polipropileno }\end{array}$ \\
\hline
\end{tabular}
la mesa como parte del análisis morfológico

\subsubsection{Objetivos ponderados}

La elección del diseño final se llevó a cabo utilizando el método de objetivos ponderados, el cual brindó la posibilidad de realizar una evaluación cuantitativa de cada uno de los cuatro bocetos propuestos (se muestran 3), lo que permitió una elección del mobiliario que supliera en mayor proporción las necesidades establecidas por los estudiantes en la Casa de la Calidad.

Dentro del método se asignó una ponderación según el cumplimiento del requerimiento, la cual se multiplicó con la importancia del requerimiento para el usuario, preliminarmente establecida. Para realizar esta valoración se tuvo en cuenta un puntaje similar al utilizado en la Casa de la Calidad al momento de evaluar el cumplimiento de los proveedores, en la cual se asigna 5 si se cumple totalmente el requerimiento, 3 si lo cumple de forma parcial, 1 si lo cumple de una forma deficiente y 0 si definiti- 
vamente no lo cumple o el diseño no funciona para esta necesidad.

Por último, se realizó una sumatoria de los datos obtenidos en cada uno de los diseños con el fin de obtener el mayor puntaje. En este caso, el diseño número 4 arrojó una puntuación de 470 , lo que indica un cumplimiento del $98 \%$ de las necesidades de los estudiantes (algunas necesidades se ven en la tabla 2).

Tabla 2. Aplicación del método de objetivos ponderados de acuerdo con las variaciones de las características del diseño del escritorio

\begin{tabular}{|c|c|c|c|c|c|c|c|}
\hline \multirow[b]{2}{*}{$\begin{array}{l}\text { Necesidad del } \\
\text { Cliente }\end{array}$} & \multirow[b]{2}{*}{$\begin{array}{l}\text { Import. } \\
\text { Cliente }\end{array}$} & \multicolumn{2}{|c|}{ Propuesta 1} & \multicolumn{2}{|c|}{ Propuesta 2} & \multicolumn{2}{|c|}{ Propuesta 4} \\
\hline & & Calificación & Valor & Calificación & Valor & Calificación & Valor \\
\hline $\begin{array}{l}\text { Amplio y } \\
\text { permite } \\
\text { acomodar los } \\
\text { elementos de } \\
\text { estudio. }\end{array}$ & 5 & 5 & 25 & 3 & 15 & 5 & 25 \\
\hline $\begin{array}{l}\text { Permite la } \\
\text { movilidad de las } \\
\text { extremidades } \\
\text { inferiores. }\end{array}$ & 5 & 5 & 25 & 3 & 15 & 5 & 25 \\
\hline $\begin{array}{l}\text { Ergonómico para } \\
\text { movilidad }\end{array}$ & 5 & 3 & 15 & 3 & 15 & 5 & 25 \\
\hline $\begin{array}{l}\text { Medidas } \\
\text { antropométricas } \\
\text { estándar }\end{array}$ & 5 & 5 & 25 & 5 & 25 & 5 & 25 \\
\hline $\begin{array}{l}\text { La ubicación de } \\
\text { la CPU permite } \\
\text { la movilidad de } \\
\text { las extremidades } \\
\text { inferiores. }\end{array}$ & 3 & 5 & 15 & 5 & 15 & 5 & 15 \\
\hline $\begin{array}{l}\text { Espacio } \\
\text { adecuado para } \\
\text { la ubicación y } \\
\text { movilidad del } \\
\text { mouse. }\end{array}$ & 3 & 3 & 9 & 3 & 9 & 5 & 15 \\
\hline $\begin{array}{l}\text { Dispositivo para } \\
\text { la ubicación de } \\
\text { las maletas. }\end{array}$ & 1 & 0 & 0 & 0 & 0 & 5 & 5 \\
\hline $\begin{array}{l}\text { Permite una } \\
\text { adecuada } \\
\text { organización del } \\
\text { cableado. }\end{array}$ & 1 & 0 & 0 & 0 & 0 & 5 & 5 \\
\hline $\begin{array}{l}\text { Contribuye a la } \\
\text { optimización del } \\
\text { espacio }\end{array}$ & 1 & 1 & 1 & 5 & 5 & 5 & 5 \\
\hline $\begin{array}{l}\text { La ubicación de } \\
\text { la CPU permite el } \\
\text { fácil acceso para } \\
\text { el uso de USB. }\end{array}$ & 1 & 5 & 5 & 5 & 5 & 5 & 5 \\
\hline TOTAL & & Diseño 1 & 378 & Diseño 2 & 383 & Diseño 4 & 470 \\
\hline
\end{tabular}

Propuesta de diseño de mobiliario para laboratorios informáticos de la Facultad de Ingeniería de la Universidad Distrital Francisco José de Caldas 


\subsection{Diseño seleccionado}

El diseño seleccionado (figura 6) satisface criterios evaluados con el método de la Casa de la Calidad, dentro de los que se encuentran los listados en la tabla 3.

Tabla 3. Algunas características del diseño seleccionado

Cuenta con las medidas establecidas en la normatividad vigente, y se adapta a las medidas antropométricas de los usuarios, lo que contribuye a su bienestar integral.

Cuenta con un sistema de ruedas con frenos, de tal manera que sea de fácil movilidad y se pueda fijar cuando sea requerido por el usuario.

La ubicación del porta-CPU genera un mayor espacio para el movimiento de las extremidades inferiores de los usuarios. Además, puede ser removida fácilmente al estar ajustada con tornillos a la parte superior de la mesa, lo que permite al mobiliario adaptarse a diferentes tipos de tecnologías.

El diseño de todo el mobiliario sugiere la aplicación de bordes redondeados que proporcionan seguridad y contribuyen a mantener el bienestar de los usuarios.

El material de elaboración de la mesa es en aglomerado, recubierto de fórmica de color mate, lo cual le brinda resistencia y permite prolongar su vida útil, además de facilitar su mantenimiento.

A pesar de que la mesa no cuenta con una altura ajustable, la silla del mobiliario cuenta con un rango de ajuste para su altura, que facilita la adaptación del usuario con el mobiliario.

El diseño cuenta con una parrilla deslizable que tiene como funcionalidad ser un soporte para la toma de apuntes.

La mesa cuenta con la adaptación de un apoyapiés que contribuye a la adopción de una adecuada postura por parte de los usuarios, lo que disminuye el riesgo de sufrir enfermedades y lesiones.

El mobiliario cuenta con un dispositivo especializado para ubicar bolsos o maletas que contribuye a la comodidad del usuario, y que le permite a su vez tener mayor espacio libre.

El diseño de la mesa cuenta con un espacio para ubicar elementos de estudio, tales como libros o similares, que liberan espacio en la mesa y contribuyen a la comodidad de los usuarios.

El diseño del mobiliario cuenta con una canaleta para las conexiones, que permite la organización del cableado, mejora la ubicación de la pantalla y facilita el acceso a las conexiones para los usuarios, lo que a su vez repercute en la disminución de riesgos de accidentes al no existir cables sueltos en el suelo, al permitir visualizar adecuadamente la pantalla y al evitar que los usuarios adopten posturas incorrectas buscando conexiones en el suelo o en la CPU.
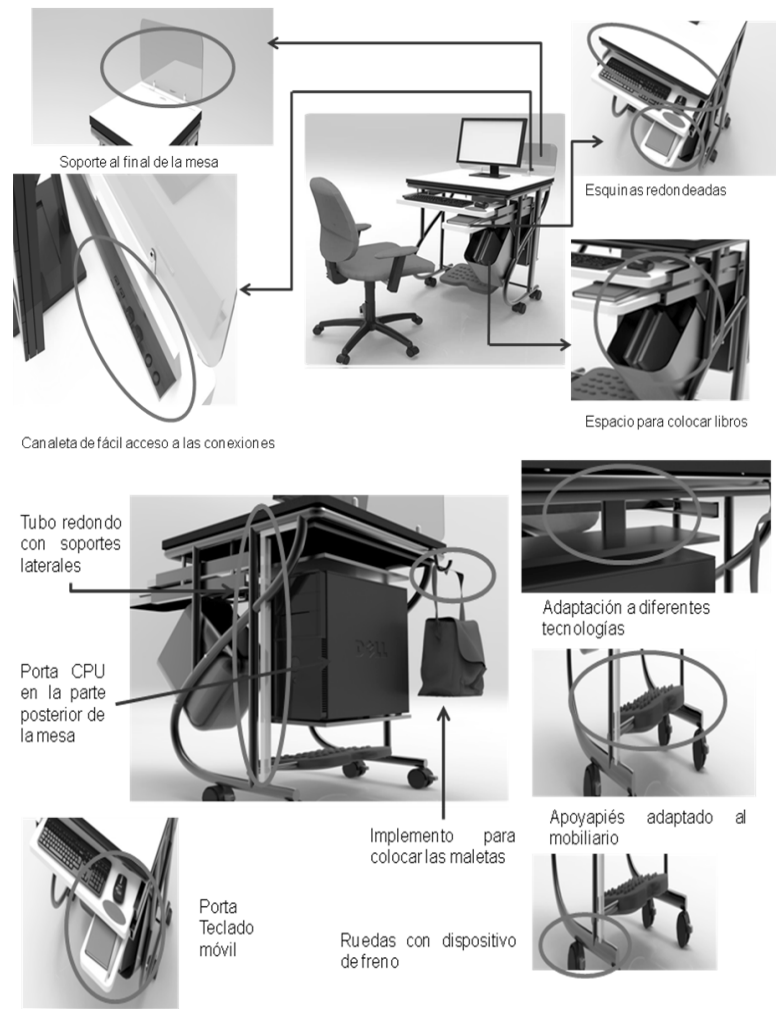

Figura 6. Diseño seleccionado

\subsection{Distribución del espacio}

Teniendo en cuenta que la distribución del espacio debe asegurar el buen uso del recurso y la movilidad adecuada del usuario en el desarrollo de actividades, se realizó una propuesta con dos alternativas. Cabe resaltar que se tomó en cuenta la NTC 4595, que establece que la distancia mínima que debe existir entre un tablero y un usuario es de $2 \mathrm{~m}$. Además, los pasillos deben contar con un ancho efectivo superior a $0,8 \mathrm{~m}$, que es el espacio necesario con el que debe contar una puerta de acceso a una sala de informática y que facilita la movilidad y desplazamiento de los usuarios.

Para la recreación del ambiente se tomó como referencia la sala 506 (existen 12 salas en total), cuya disposición es la más común en las salas de informática. De ella surgieron las opciones de las figuras $8 \mathrm{a}$ y $8 \mathrm{~b}$. Sin embargo, se elige la opción de la figura 8 a, ya que no sólo permite una adecuada movilidad y se adapta al cupo actual de estudiantes en este tipo de prác- 
ticas, sino que también se les brinda unas mejores condiciones de trabajo, tanto por el diseño del mobiliario como por su ubicación en el espacio, la cual les permite a todos ver el tablero, aspecto fundamental para el desarrollo de sus actividades académicas.

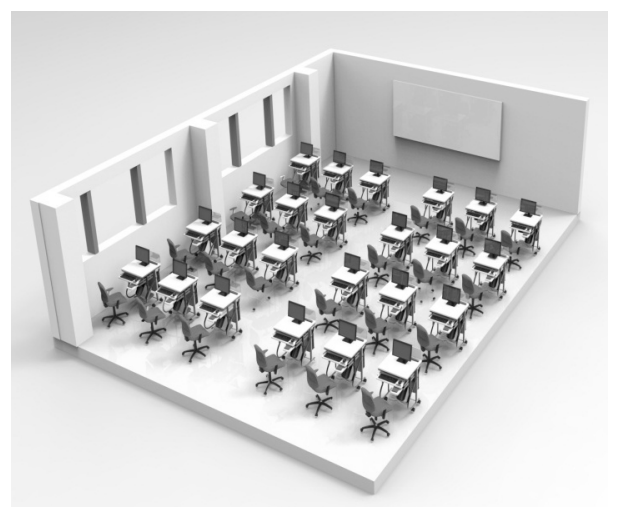

Figura 8a. Distribución propuesta manteniendo cantidad estudiantes

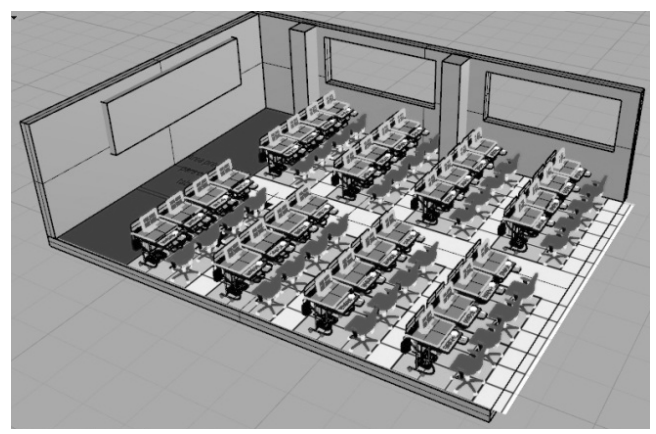

Figura 4b. Distribución propuesta aumentando cupo de estudiantes

\section{Referencias}

[1] F. Llaneza Álvarez, Ergonomía y psicosociología aplicada. Manual para la formación del especialista, $13^{\mathrm{a}}$ ed. España: Lexnova, 2009.

[2] J, Estrada. Ergonomía, $2^{\text {a }}$ ed. Medellín: Editorial Universidad de Antioquia, 2000.

[3] Jorge Acuña, Mejoramiento de la calidad, un enfoque a los servicios, $1^{\mathrm{a}}$ ed. Editorial Tecnológica de Costa Rica, 2005.

[4] J. De la Iglesia., F. Martín y E. Yacuzzi. El método de Kano en el diseño de productos

\section{CONCLUSIONES}

Es importante enfatizar que una de las ventajas de la Casa de la Calidad es la eliminación de la subjetividad durante su desarrollo. Es decir, que cada decisión está atada a un método que la aparta de lo subjetivo, como el análisis morfológico para la toma de decisión del boceto elegido y/o el método de ponderación para determinar el nivel de relación que existe entre las características que se evalúan en las matrices de la casa de la calidad.

El mobiliario que actualmente se encuentra en los laboratorios no se adapta a las herramientas tecnológicas actuales para las que es empleado, no ha sido elaborado completamente ajustado a la normatividad vigente, ni a las condiciones antropométricas de los usuarios. En contraste, el mobiliario propuesto cumple con los requerimientos realizados por los usuarios, además de responder a la normatividad vigente, los requerimientos antropométricos, los aspectos ergonómicos y de salud ocupacional y las características técnicas en general que se determinaron en el desarrollo de este proyecto.

El mobiliario propuesto permite una mejor distribución del espacio disponible, con lo que se logra mejorar la comodidad de los usuarios y facilitar actividades como ver. D. Goetsch. Quality Function Deployment. México. Editorial Merrill, 2000

[6] K. Ulrich. Diseño y desarrollo de productos, $3^{\mathrm{a}}$ Ed. México, Editorial McGraw Hill 2004.

[7] D. Winter. Biomechanics and Motor Control of Human Movement, $4^{\mathrm{a}}$ Ed. New York. John Wiley \& Sons Inc., 2009.

[8] P. Grima y J. Tort. Técnicas para la gestión de la Calidad. Madrid: Ediciones

Propuesta de diseño de mobiliario para laboratorios informáticos de la Facultad de Ingeniería de la Universidad Distrital Francisco José de Caldas 
Díaz de Santos, 1995.

[9] F. Philip Rice. Desarrollo Humano, Estudio del ciclo vital. $2^{\text {a }}$ Ed. Barcelona. Pearson Educación, 1997.

[10] M. Carl y R. Gates. Investigación de mercados, $6^{\mathrm{a}}$ Ed. México: Thomson, 2005.

[11] F. Velázquez. Manual de ergonomía. Espalña: Editorial Mapfre, S.A., 1995.

[12] Muebles Carvajal (2007), [en línea]. Consultado en febrero de 2013, disponible en: http://www.mepal.co//link
[13] Universidad Jorge Tadeo Lozano (2005), [en línea]. Consultado en febrero de 2013, disponible en http://www.solinoff.com/ producto/proyectos/sector-institucional/ univesidad-jorge-tadeo-lozano/37/37/201

[14] Servex Colombia (2005), [en línea]. Consultado en febrero de 2013, disponible en http://www.servexcolombia.com/ html5/content.php?pagename $=$ Easy 